\title{
A Criterion for the Anisotropy Barrier in Single-Molecule Magnets
}

\author{
Oliver Waldmann* \\ Department of Chemistry and Biochemistry, University of Bern, CH-3012 Bern, Switzerland
}

\section{Supporting Information}

The derivation of the lower and upper bounds for the projection coefficients $d_{i}$, and therewith of the criterion Eq. (8), shall be presented in some detail. At the end the key ideas are summarized in an easy to comprehend fashion.

The anisotropy barrier $\Delta=|D| S^{2}$ is considered for a single-molecule magnet (SMM), whose magnetism (in zero magnetic field) can be well described by the microscopic spin Hamiltonian

$$
\begin{aligned}
& \hat{H}=-\sum_{i \neq j} J_{i j} \hat{\mathbf{S}}_{i} \cdot \hat{\mathbf{S}}_{j}+\hat{H}_{1}, \\
& \hat{H}_{1}=\sum_{i} \hat{\mathbf{S}}_{i} \cdot \mathbf{D}_{i} \cdot \hat{\mathbf{S}}_{i}+\sum_{i \neq j} \hat{\mathbf{S}}_{i} \cdot \mathbf{D}_{i j}^{d i p} \cdot \hat{\mathbf{S}}_{j}+\hat{H}^{\prime},
\end{aligned}
$$

consisting of the Heisenberg exchange interactions, the local zero-field-splitting (ZFS) terms due to the ligand-field interactions, the dipole-dipole interactions, and further terms, which are collected in $\hat{H}^{\prime}$ ( $\hat{\mathbf{S}}_{i}$ is the spin operator on site $i$ with length $S_{i}$, the number of spin centers in the cluster will be denoted as $N$ ). The Heisenberg term is assumed to be the dominant term, and $\hat{H}_{1}$ is considered a perturbation. The energy spectrum of the Heisenberg term consists of spin multiplets, which can be characterized by the total spin quantum number $S$ and magnetic quantum number $M$; the eigenfunctions will be denoted as $|\alpha S M\rangle$, where $\alpha$ stands for all additional quantum numbers required for an unambiguous labeling. A particular spin multiplet or eigenstate, respectively, is hence identified through the labels $\alpha$ and $S$.

The perturbation by $\hat{H}_{1}$ leads to a ZFS of each spin multiplet, which can be described by an effective spin Hamiltonian $\hat{H}_{\alpha S}$. In the strong-exchange limit, or in first-order perturbation theory in $\hat{H}_{1}$, respectively, and neglecting $\hat{H}^{\prime}$ (which contains terms generally not important), one finds

$$
\begin{aligned}
& \hat{H}_{\alpha S}=\hat{\mathbf{S}} \cdot \mathbf{D} \cdot \hat{\mathbf{S}}, \\
& \mathbf{D}=\sum_{i} d_{i} \mathbf{D}_{i}+\sum_{i \neq j} d_{i j} \mathbf{D}_{i j}^{d i p},
\end{aligned}
$$

with the so-called projection coefficients $d_{i}$ and $d_{i j}$ (which are real scalar numbers). The tensor D characterizes the ZFS of the spin multiplet $\alpha S$ under consideration. Obviously, the ZFS will be different for different spin multiplets, and the tensor D or coefficients $d_{i}$ and $d_{i j}$, respectively, hence depend on the labels $\alpha$ and $S$ (indexing the $d$ 's with these labels is not done here for convenience). The values of the projection coefficients are determined such that the ZFS of the spin multiplet $\alpha S$ coincides with that calculated from $\hat{H}_{\alpha s}$ in first-order, as expressed by the condition

$$
\left\langle\alpha S M^{\prime}\left|\hat{H}_{1}\right| \alpha S M\right\rangle=\left\langle S M^{\prime}\left|\hat{H}_{\alpha S}\right| S M\right\rangle
$$

i.e., such that $\hat{H}_{1}$ and $\hat{H}_{\alpha S}$ are operator equivalents in the subspace of the considered spin multiplet. It is to be noted that $|\alpha S M\rangle$ denotes a spin wave function in the many-spin space of the microscopic spin Hamiltonian, while $|S M\rangle$ denotes a spin wave function of a single spin of length $S$. The actual calculation of the projection coefficients may be done in various ways. 
One approach, which is probably the most cited one, is to use the formalism of the irreducible tensor operators (ITOs) (which so to say allows one to cancel the dependence on $M^{\prime}$ and $M$ ). This yields relations between reduced matrix elements, such as

$$
\left\langle\alpha S\left\|\hat{H}_{1}\right\| \alpha S\right\rangle=\left\langle S\left\|\hat{H}_{\alpha S}\right\| S\right\rangle
$$

or, for the projection coefficients,

$$
d_{i}=\frac{\left\langle\alpha S\left\|T^{2}\left(S_{i}\right)\right\| \alpha S\right\rangle}{\left\langle S\left\|T^{2}(S)\right\| S\right\rangle}, \quad d_{i j}=\frac{\left\langle\alpha S\left\|T^{2}\left(S_{i} S_{j}\right)\right\| \alpha S\right\rangle}{\left\langle S\left\|T^{2}(S)\right\| S\right\rangle} .
$$

The ITO approach, however, is not convenient for the present purpose. An alternative technique is to use Eq. (S5) directly for a specific choice of $M^{\prime}$ and $M$, for instance, $M^{\prime}=M=S$. Furthermore, since the values of the projection coefficients do not depend on the particular form of the tensors $\mathbf{D}_{i}$ and $\mathbf{D}_{i j}^{\text {dip }}$, they may be chosen at will, e.g., as diag(-1,-1,2), such that

$$
\begin{aligned}
& \hat{\mathbf{S}}_{i} \cdot \mathbf{D}_{i} \cdot \hat{\mathbf{S}}_{i} \rightarrow 3 \hat{S}_{i, z}^{2}-S_{i}\left(S_{i}+1\right), \\
& \hat{\mathbf{S}}_{i} \cdot \mathbf{D}_{i j} \cdot \hat{\mathbf{S}}_{j} \rightarrow 2 \hat{S}_{i, z} \hat{S}_{j, z}-\hat{S}_{i, x} \hat{S}_{j, x}-\hat{S}_{i, y} \hat{S}_{j, y}, \\
& \hat{\mathbf{S}} \cdot \mathbf{D} \cdot \hat{\mathbf{S}} \rightarrow 3 \hat{S}_{z}^{2}-S(S+1) .
\end{aligned}
$$

The projection coefficients are then determined by the relations

$$
\begin{aligned}
& \left\langle\alpha S S\left|3 \hat{S}_{i, z}^{2}-S_{i}\left(S_{i}+1\right)\right| \alpha S S\right\rangle=d_{i}\left\langle S S\left|3 \hat{S}_{z}^{2}-S(S+1)\right| S S\right\rangle, \\
& \left\langle\alpha S S\left|2 \hat{S}_{i, z} \hat{S}_{j, z}-\hat{S}_{i, x} \hat{S}_{j, x}-\hat{S}_{i, y} \hat{S}_{j, y}\right| \alpha S S\right\rangle=d_{i j}\left\langle S S\left|3 \hat{S}_{z}^{2}-S(S+1)\right| S S\right\rangle .
\end{aligned}
$$

The matrix element on the right side is easily calculated to $\left\langle S S\left|3 \hat{S}_{z}^{2}-S(S+1)\right| S S\right\rangle=S(2 S-1)$. The evaluation of the matrix elements on the left side, however, requires the detailed knowledge of the wave function $|\alpha S S\rangle$. It will be expanded below in terms of the product wave functions (= uncoupled basis)

$$
|\gamma\rangle \equiv \prod_{i}\left|S_{i}, M_{i}\right\rangle=\left|M_{1} M_{2} \ldots M_{N}\right\rangle .
$$

For the ferromagnetic spin multiplet, for which $S$ assumes the maximal value

$$
S_{\max }=\sum_{i} S_{i}
$$

the wave function with $M=S$ is simply the Ising state with all spins “up”, i.e., the product wave function

$$
|F\rangle \equiv \prod_{i}\left|S_{i}, S_{i}\right\rangle=\left|S_{1} S_{2} \ldots S_{N}\right\rangle
$$

For the wave function $|F\rangle$ the calculation of the matrix elements is trivial,

$$
\begin{aligned}
& \left\langle F\left|3 \hat{S}_{i, z}^{2}-S_{i}\left(S_{i}+1\right)\right| F\right\rangle=S_{i}\left(2 S_{i}-1\right) \\
& \left\langle F\left|2 \hat{S}_{i, z} \hat{S}_{j, z}-\hat{S}_{i, x} \hat{S}_{j, x}-\hat{S}_{i, y} \hat{S}_{j, y}\right| F\right\rangle=2 S_{i} S_{j}
\end{aligned}
$$

which immediately yields

$$
d_{i}^{F}=\frac{S_{i}\left(2 S_{i}-1\right)}{S(2 S-1)}, \quad d_{i j}^{F}=\frac{2 S_{i} S_{j}}{S(2 S-1)},
$$

(a subscript $F$ has been added to the $d$ 's for obvious reasons). 
For a general spin multiplet, with $S \leq S_{\max }$, lower and upper bounds can be derived for the projection coefficients $d_{i}$ of the local ZFS. Since obviously $\left\langle\alpha S S\left|S_{i}\left(S_{i}+1\right)\right| \alpha S S\right\rangle=S_{i}\left(S_{i}+1\right)$, one has to consider the matrix element $\left\langle\alpha S S\left|\hat{S}_{i, z}^{2}\right| \alpha S S\right\rangle$. Expanding the wave function $|\alpha S S\rangle$ in terms of the product wave functions,

$$
|\alpha S S\rangle=\sum_{\gamma} c_{\gamma}|\lambda\rangle
$$

yields

$$
\left\langle\alpha S S\left|\hat{S}_{i, z}^{2}\right| \alpha S S\right\rangle=\sum_{\gamma^{\prime}} c_{\gamma}^{*} c_{\gamma^{\prime}}\left\langle\gamma\left|\hat{S}_{i, z}^{2}\right| \gamma^{\prime}\right\rangle=\sum_{\gamma} c_{\gamma}^{2}\left\langle\gamma\left|\hat{S}_{i, z}^{2}\right| \gamma\right\rangle
$$

It is now convenient to change the indexing of the product wave functions such that all wave functions with equal value of $M_{i}$ are labeled by $M_{i}$ and a further index $\beta$, i.e., to write $\gamma \rightarrow \beta M_{i}$ and $\sum_{\gamma} \rightarrow \sum_{M_{i}} \sum_{\beta}$. One then obtains

$$
\left\langle\alpha S S\left|\hat{S}_{i, z}^{2}\right| \alpha S S\right\rangle=\sum_{M_{i}} \sum_{\beta} c_{\beta M_{i}}^{2}\left\langle\beta M_{i}\left|\hat{S}_{i, z}^{2}\right| \beta M_{i}\right\rangle=\sum_{M_{i}} \sum_{\beta} c_{\beta M_{i}}^{2} M_{i}^{2}=\sum_{M_{i}} c_{M_{i}}^{2} M_{i}^{2}
$$

with $c_{M_{i}}^{2}=\sum_{\beta} c_{\beta M_{i}}^{2}$. The maximal (minimal) value of $\left\langle\alpha S S\left|\hat{S}_{i, z}^{2}\right| \alpha S S\right\rangle$ may be hence found from maximizing (minimizing) the sum $\sum_{M_{i}} c_{M_{i}}^{2} M_{i}^{2}$ with the constraints $\sum_{M_{i}} c_{M_{i}}^{2}=1$ and $0 \leq c_{M_{i}}^{2} \leq 1$. The maximum may be derived as follows. With the choice $c_{M_{i}}^{2}=1$ for $M_{i}=S_{i}$ and $c_{M_{i}}^{2}=0$ for $M_{i} \neq S_{i}$, one finds $\sum_{M_{i}} c_{M_{i}}^{2} M_{i}^{2}=S_{i}^{2}$. This is already the maximum as can be inferred from the choice $c_{M_{i}}^{2}=c$ with $c<1$ for $M_{i}=S_{i}, c_{M_{i}}^{2}=1-c$ for an arbitrary $M_{i}$ with $\left|M_{i}\right|<S_{i}$, and $c_{M_{i}}^{2}=0$ for all other $M_{i}$, which gives $\sum_{M_{i}} c_{M_{i}}^{2} M_{i}^{2}=c S_{i}^{2}+(1-c) M_{i}^{2}<c S_{i}^{2}+(1-c) S_{i}^{2}=S_{i}^{2}$. One hence finds $\sum_{M_{i}} c_{M_{i}}^{2} M_{i}^{2} \leq S_{i}^{2}$. In a similar way one can find the minimal value of $\sum_{M_{i}} c_{M_{i}}^{2} M_{i}^{2}$. For integer $S_{i}$, the smallest value of $M_{i}$ is zero, hence $0 \leq \sum_{M_{i}} c_{M_{i}}^{2} M_{i}^{2}$ (the minimum is attained for $c_{M_{i}=0}^{2}=1$ ); for half-integer $S_{i}$, the smallest value of $\left|M_{i}\right|$ is $1 / 2$, hence $1 / 4 \leq \sum_{M_{i}} c_{M_{i}}^{2} M_{i}^{2}$ (the minimum is attained for $c_{M_{i}= \pm 1 / 2}^{2}=1$ ). Putting all together one finds

$$
\left.\begin{array}{cc}
-S_{i}\left(S_{i}+1\right) & \forall \text { integer } S_{i} \\
3 / 4-S_{i}\left(S_{i}+1\right) & \forall \text { half -integer } S_{i}
\end{array}\right\} \leq\left\langle\alpha S S\left|3 \hat{S}_{i, z}^{2}-S_{i}\left(S_{i}+1\right)\right| \alpha S S\right\rangle \leq S_{i}\left(2 S_{i}-1\right)
$$

Table 1 in the manuscript shows that, with the exception of $S_{i}=1$, the magnitude of the lower bound is always equal to or smaller than the upper bound, such that one can further simplify to

$$
\left|\left\langle\alpha S S\left|3 \hat{S}_{i, z}^{2}-S_{i}\left(S_{i}+1\right)\right| \alpha S S\right\rangle\right| \leq S_{i}\left(2 S_{i}-1\right) \quad \forall S_{i}>1
$$

or equivalently to

$$
\left|d_{i}\right| \leq \frac{S_{i}\left(2 S_{i}-1\right)}{S(2 S-1)} \quad \forall S_{i}>1
$$

For $S_{i}=1$, the magnitude of the lower bound is twice as large as the upper bound, such that an additional factor of 2 appears, i.e.,

$$
\left|d_{i}\right| \leq 2 \frac{S_{i}\left(2 S_{i}-1\right)}{S(2 S-1)} \quad \forall S_{i}=1
$$

It is convenient to introduce the "local anisotropy barrier" of the $i$-th spin center,

$$
\Delta_{i}=\left\{\begin{array}{ll}
2\left|D_{i}\right| S_{i}^{2} & \forall S_{i}=1 \\
\left|D_{i}\right| S_{i}^{2} & \forall S_{i}>1
\end{array},\right.
$$

(the factor of 2 in the case of $S_{i}=1$ is hence accounted for through the definition of the local anisotropy barrier). The contribution $d_{i}\left|D_{i}\right|$ of the $i$-th spin center to the anisotropy barrier $\Delta$ (of the whole cluster) is bounded as 


$$
\left|d_{i}\right| D_{i}|| \leq \frac{\left(2-1 / S_{i}\right)}{S^{2}(2-1 / S)} \Delta_{i}
$$

Ignoring the dipole-dipole contribution, Eq. (S4) implies for the anisotropy barrier of the cluster

$$
\Delta=|D| S^{2}=\left|\sum_{i=1}^{N} d_{i} \mathbf{D}_{i}\right| S^{2} \leq \sum_{i=1}^{N}\left|d_{i}\right| D_{i} \mid S^{2}
$$

and inserting Eq. (S16) leads to $\Delta \leq \Delta_{\max }$ with

$$
\Delta_{\max }=\sum_{i=1}^{N} \frac{\left(2-1 / S_{i}\right)}{(2-1 / S)} \Delta_{i}
$$

Further considering that $\left(2-1 / S_{i}\right) /(2-1 / S) \leq 1$ for $S \geq S_{i}$ yields $\Delta_{\max } \leq \sum_{i} \Delta_{i}$, such that one finally obtains

$$
\Delta \leq \Delta_{\max } \leq \sum_{i=1}^{N} \Delta_{i}
$$

Equations (S12) to (S18) are the main result of this work. They show that the cluster anisotropy $|D|$ scales with $S$ as $S^{-2}$, such that the anisotropy barrier $\Delta=|D| S^{2}$ scales as $S^{0}$, i.e., is largely independent on $S$ !

For the projection coefficient $d_{i j}$ of the dipole-dipole interactions lower and upper bounds are not as easily derived. However, considering the calculation for the local ZFS and the nature of the problem, it is clear that the magnitude of the matrix element $\left\langle\alpha S S\left|2 \hat{S}_{i, z} \hat{S}_{j, z}-\hat{S}_{i, x} \hat{S}_{j, x}-\hat{S}_{i, y} \hat{S}_{j, y}\right| \alpha S S\right\rangle$ has to be on the order of $2 S_{i} S_{j}$, that is

$$
\left|\left\langle\alpha S S\left|2 \hat{S}_{i, z} \hat{S}_{j, z}-\hat{S}_{i, x} \hat{S}_{j, x}-\hat{S}_{i, y} \hat{S}_{j, y}\right| \alpha S S\right\rangle\right| \leq 2 c S_{i} S_{j}
$$

with some suitable constant $c$ of order 1 ( $c$ is independent on other parameters, such as the number $N$ of metal centers). Following the procedure as for the local ZFS gives the bound $2 S_{i} S_{j}+\sqrt{S_{i}\left(S_{i}+1\right)} \sqrt{S_{j}\left(S_{j}+1\right)}$, i.e., $c \approx 1.5$, but it might well be that a more careful calculation would produce a tighter bound. Anyhow, in any case, also the $d_{i j}$ scale with $S$ as $S^{-2}$, such that the general conclusions drawn from considering only the local ZFS remain unaffected by the inclusion of the dipole-dipole interactions. Furthermore, although the dipole-dipole interactions are known to contribute substantially to the cluster ZFS in some cases (e.g., in Fe(III) clusters they may be responsible for some few 10\%), they should be of less importance for SMMs in general, since for these clusters one tends to prefer magnetic metal ions providing large local ZFS.

\section{Summary:}

Within the strong-exchange limit of the microscopic spin Hamiltonian, Eqs. (S1) and (S2), tuning the anisotropy barrier is achieved by controlling the exchange-coupling constants $J_{i j}$ and the local ZFS tensors $\mathbf{D}_{i}$ (the dipole-dipole interactions are again ignored for the clarity of the argument). The exchange-coupling constants determine the ground-state multiplet, $|\alpha S M\rangle$, and wherewith BOTH the value of $S$ and the values of the projection coefficients $d_{i}$; $S$ and the $d_{i}$ are so to say functions of the $J_{i j}$ (but do NOT depend on the $\mathbf{D}_{i}$ ). The cluster ZFS is related to the local ZFS tensors via $\mathbf{D}=\sum_{i} d_{i} \mathbf{D}_{i}$, which involves also the projections coefficients. For the projection coefficients one has the general result that they scale with $S$ as $S^{-2}$; hence also $\mathbf{D}$ and its magnitude $|D|$, respectively, scale as $S^{-2}$, such that the anisotropy barrier $\Delta=|D| S^{2}$ scales as $S^{0}$. We can summarize:

$$
\begin{aligned}
& -\sum_{i \neq j} J_{i j} \hat{\mathbf{S}}_{i} \cdot \hat{\mathbf{S}}_{j} \rightarrow S, d_{i} \\
& d_{i} \propto \frac{1}{S^{2}} \rightarrow|D| \propto \frac{1}{S^{2}} \rightarrow \Delta=|D| S^{2} \propto S^{0}
\end{aligned}
$$

This is all this work wishes to point out. 